\title{
A tool to balance benefit and harm when deciding about adjuvant therapy
}

\author{
AM Knops ${ }^{*, 1}$, A Goossens', MPM Burger², LJA Stalpers ${ }^{3}$ and DT Ubbink' \\ 'Department of Quality Assurance and Process Innovation, Academic Medical Center at the University of Amsterdam, The Netherlands; ${ }^{2}$ Department of \\ Gynaecology, Academic Medical Center at the University of Amsterdam, The Netherlands; ${ }^{3}$ Department of Radiotherapy, Academic Medical Center at \\ the University of Amsterdam, The Netherlands
}

Adjuvant therapy aims to prevent outgrowth of residual disease but can induce serious side effects. Weighing conflicting treatment effects and communicating this information with patients is not elementary. This study presents a scheme balancing benefit and harm of adjuvant therapy vs no adjuvant therapy. It is illustrated by the available evidence on adjuvant pelvic external beam radiotherapy (RT) for intermediate-risk stage I endometrial carcinoma patients. The scheme comprises five outcome possibilities of adjuvant therapy: patients who benefit from adjuvant therapy (some at the cost of complications) vs those who neither benefit nor contract complications, those who do not benefit but contract severe complications, or those who die. Using absolute risk differences, a fictive cohort of 1000 patients receiving adjuvant RT is categorised. Three large randomised clinical trials were included. Recurrences will be prevented by adjuvant RT in 60 patients, a majority of 908 patients will neither benefit nor suffer severe radiation-induced harm but 28 patients will suffer severe complications due to adjuvant RT and an expected four patients will die. This scheme readily summarises the different possible treatment outcomes and can be of practical value for clinicians and patients in decision making about adjuvant therapies.

British Journal of Cancer (2009) 100, 913-917. doi:10.1038/sj.bjc.6604962 www.bjcancer.com (C) 2009 Cancer Research UK

Keywords: absolute risk increase; absolute risk reduction; adjuvant radiotherapy; decision making; endometrial carcinoma

Adjuvant therapy aims to prevent outgrowth of residual disease after surgical removal of a malignancy to improve disease-specific and overall survival. However, adjuvant therapy can induce serious side effects as well. Therefore, the benefit of adjuvant therapy in terms of a reduced risk of recurrence needs to be weighed carefully against the risk of therapy-induced harm, before a decision is made.

Particularly when the effect of available treatment options is ambiguous or equal, patient preferences can play a crucial role in decision making. In a recent study by Pieterse et al (2007), the deciding benefit to choose for neoadjuvant radiotherapy (RT) and the importance of different treatment outcomes were found to vary widely among rectal cancer patients as well as between oncologists and patients. Moreover, oncology patients were found to be more than willing to participate in decision making after being thoroughly informed (van Tol-Geerdink et al, 2006). Clinicians should therefore adequately inform their patients about the risks and benefits of adjuvant therapy, and elicit patient preferences regarding treatment outcomes.

It is not elementary to weigh conflicting treatment effects and to communicate this information with patients. Parameters such as

\footnotetext{
* Correspondence: AM Knops, Department of Quality Assurance and Process Innovation, Academic Medical Center, Room JI B 207. I, PO Box 22700, I 100 DE Amsterdam, The Netherlands;

E-mail: a.m.knops@amc.nl

Received 14 October 2008; revised 9 February 2009; accepted 10 February 2009
}

number needed to treat (NNT) and number needed to harm (NNH) have been proposed to give the clinician some direction of the benefit and harm of adjuvant therapies. However, they often over-simplify the consequences of a treatment (Nuovo et al, 2002; Massel, 2003). For example, NNT does not differentiate between patients in whom a recurrence is prevented without suffering side effects and patients who are spared from a recurrence but who do suffer serious side effects. Thus, these rather intangible numbers cannot express how many patients will benefit or suffer from the adjuvant therapy.

In intermediate-risk stage I endometrial carcinoma, for instance, adjuvant pelvic external beam RT is presently the acknowledged treatment to reduce the risk of outgrowth of residual disease in the pelvis (http://www.guideline.gov/summary/summary.aspx?doc_id = 9203\&nbr $=004957 \&$ string $=$ endometrial+AND+cancer). Nevertheless, a great international controversy exists (Burger, 2001; Johnson and Cornes, 2007). In a recent meta-analysis, the benefits of adjuvant RT were reviewed. In the subgroup of intermediate-risk patients, a statistically significant reduction of local recurrences was observed when adjuvant RT was applied compared with no adjuvant therapy following surgery (NNT 16.7 women; $95 \%$ confidence interval $12.5-$ 25.0). However, this advantage did not result in a better overall or endometrial carcinoma-related survival for these patients (Kong et al, 2007). No clear recommendations for clinical practice were given based on these results.

A plain scheme presenting (the risk of) all possible outcomes of adjuvant therapy could provide more insights for clinicians as well as for patients. Such a tool has been designed earlier with respect to neoadjuvant RT for resectable rectal 
carcinomas (Bakx et al, 2006). Yet, this has not been recognised as a useful tool to facilitate clinician-patient communication when vacillating about the decision for (neo)adjuvant cancer therapy.

The aim of this study is to present a scheme balancing the benefit and harm of adjuvant therapy $v s$ no adjuvant therapy, illustrated by the available evidence on adjuvant pelvic external beam RT for intermediate-risk stage I endometrial carcinoma patients.

\section{MATERIALS AND METHODS}

The scheme comprises five outcome possibilities of adjuvant therapy, ranging from patients experiencing full benefit without any harm to patients experiencing full harm, that is death from adjuvant therapy (Table 1). Presenting these different outcome possibilities in natural frequencies is not yet very common, but helpful (Hoffrage et al, 2000). Therefore, evidence on the beneficial and harmful effects of adjuvant RT was needed to enter data into the scheme.

All available relevant evidence was searched in PubMed, and studies were included if they comprised (1) randomised clinical trials (RCTs), (2) comparing adjuvant pelvic external beam RT following surgery $v s$ surgery alone and (3) in women with stage 1 intermediaterisk endometrial carcinoma. Local recurrences and RT-induced harm had to be one of the clinical end-points measured.

The extent of benefit and harm in the included studies was determined by means of the absolute risk reduction (ARR) and absolute risk increase (ARI) (McAlister et al, 2000; Straus, 2002). Treatment benefit can be expressed as an ARR. This expresses the absolute additional beneficial effect of adjuvant therapy over no adjuvant treatment and is equal to the difference between control event rate and experimental event rate found in RCTs (ARR = event rate control group-event rate intervention group). The ARR can also be calculated as the reciprocal of NNT, which is commonly used to express the number of patients who needs to be treated to gain one additional beneficial outcome. For instance, an NNT of 5 corresponds to an ARR of $1 / 5=0.2$.

Although survival is considered to be the ultimate primary outcome in oncology trials, the available RCTs considering adjuvant RT in stage I intermediate-risk endometrial cancer patients have shown neither overall survival benefit nor endometrialcarcinoma-related survival advantage. Hence, in this analysis, we chose local recurrence rate $\left(\mathrm{ARR}_{\text {local recurrence }}\right)$ as the primary outcome parameter. Preventing local recurrences is relevant if it increases the rate of cancers cured and if it avoids the women's mental and physical morbidity of additional diagnosis and treatment (Creutzberg et al, 2000).

The ARI is a measure of treatment-induced harm. It is defined by the absolute difference in complication rates between two treatment modalities (ARI = complication rate intervention groupcomplication rate control group). The ARI can also be calculated as the reciprocal of $\mathrm{NNH}$, which is used to express the number of patients who has to undergo treatment to induce one additional harmful event. In this analysis, the ARI was the difference between the complication rates of women with and without adjuvant RT. Complication rates involved morbidity and mortality, resulting in $\mathrm{ARI}_{\text {morbidity }}$ and ARI $\mathrm{Amortality}_{\text {. }}$

Although mortality is a clear-cut end-point, morbidity may be subject to discussion regarding the level of severity. In this analysis, only severe grade 3 complications, according to the French - Italian glossary, were taken into account (Chassagne et al, 1993), because the severity of these complications could cause women to reconsider their decision about having adjuvant RT. Grade 3 complications are life-threatening per se or due to their treatment required. They also comprise any permanent and severe tissue or organ damage (e.g., stenosis, bleeding, or fistulas of the intestines or of the urinary tract). Grade 4 complications are deaths due to a complication of the treatment of cancer, comprising ARI $\mathrm{Aortality}_{\text {. }}$

From the included studies, the reported number of study participants in the RT and control groups, the number of local recurrences, severe complications and deaths due to adjuvant RT were extracted (Table 2). These were combined into an overall ARR, overall ARI for morbidity and overall ARI for mortality. A 95\% CI was determined to provide an estimate of the precision of the ARR and ARIs.

Subsequently, patients receiving adjuvant RT were divided into five outcome groups based on the benefit and harm the therapy may induce. As using absolute numbers and stating the reference class is likely to improve the understanding of risks and benefits (Gigerenzer and Edwards, 2003), this is illustrated in a fictive cohort of 1000 women receiving adjuvant RT. It is worth noting that the 'benefit' in this analysis is about the difference in local recurrence rates between the two treatment modalities, whereas 'harm' in this scheme concerns the difference in complication rates. Table 1 gives the equations to calculate each of the five groups (Schulzer and Mancini, 1996; Mancini and Schulzer, 1999).

\section{RESULTS}

Six RCTs, studying adjuvant pelvic external beam RT $v s$ no adjuvant therapy in early-stage endometrial carcinoma, were identified (Aalders et al, 1980; Weigensberg, 1984; Creutzberg et al, 2000; Soderini et al, 2003; Keys et al, 2004; the ASTEC/EN.5 writing committee 2008, 2009). One RCT was excluded because the control group underwent another adjuvant intervention instead of no intervention (Aalders et al, 1980). The clinical trial of Weigensberg (1984) focussed on preoperative RT (Weigensberg, 1984). The study of Soderini et al (2003) (published as an abstract only) did not report radiation-induced harm, and was therefore also excluded from analysis (Soderini et al, 2003).

Three large RCTs remained: the Post Operative Radiation Therapy in Endometrial Carcinoma-1 study from Creutzberg (PORTEC-1), the Gynecologic Oncology Group 99 study from Keys (GOG 99) and A Study in the Treatment of Endometrial Cancer/EN.5 study from the ASTEC/EN.5 writing committee 2008

Table I Five groups of possible outcomes after adjuvant RT

\begin{tabular}{|c|c|c|}
\hline Group & Definition in this analysis & Equation \\
\hline I: Full benefit, no harm & $\begin{array}{l}\text { Patients in whom RT prevented the development of a local recurrence and who do not suffer } \\
\text { from morbidity due to RT }\end{array}$ & $A R R \times\left(I-A R I_{\text {morbidity }}\right)$ \\
\hline 2: Benefit with harm & $\begin{array}{l}\text { Patients in whom RT prevented the development of a local recurrence, but suffer from } \\
\text { morbidity induced by RT }\end{array}$ & $A R R \times A R I_{\text {morbidity }}$ \\
\hline $\begin{array}{l}\text { 3: Neither benefit } \\
\text { nor harm }\end{array}$ & $\begin{array}{l}\text { Patients in whom RT did not prevent a local recurrence, but do not suffer from RT-related } \\
\text { morbidity }\end{array}$ & $(I-A R R) \times\left(I-\left(A R I_{\text {morbidity }}+A R I_{\text {mortality }}\right)\right)$ \\
\hline 4: No benefit but harm & Patients in whom RT did not prevent a local recurrence, and suffer from morbidity due to RT & $(I-A R R) \times A R I_{\text {morbidity }}$ \\
\hline 5: Full harm & Patients who die as a result of RT & $A R I_{\text {mortality }}$ \\
\hline
\end{tabular}

$A R I_{\text {morbidity }}=$ absolute risk increase in radiation-induced morbidity; $A R I_{\text {mortality }}$ absolute risk increase in radiation-induced mortality; $A R R=$ absolute risk reduction in the development of local recurrences; RT = radiotherapy. 
Table 2 Results of the included trials and overall risk differences

\begin{tabular}{|c|c|c|c|c|}
\hline & $n$ & Local recurrences (\%) & Severe morbidity (\%) & Mortality (\%) \\
\hline \multicolumn{5}{|l|}{ PORTEC-I } \\
\hline Radiotherapy & 354 & II (0.03) & $6(0.02)$ & I (0.003) \\
\hline Control & 360 & $40(0.11)$ & I (0.003) & $0(0.00)$ \\
\hline \multicolumn{5}{|l|}{ GOG 99} \\
\hline Radiotherapy & 190 & $3(0.02)$ & $9(0.05)$ & $2(0.01)$ \\
\hline Control & 202 & $18(0.09)$ & ( $(0.005)$ & $0(0.00)$ \\
\hline \multicolumn{5}{|l|}{ ASTEC/EN.5 } \\
\hline Radiotherapy & 452 & $13(0.03)$ & $34(0.08)$ & I (0.002) \\
\hline Control & 453 & $29(0.06)$ & $15(0.03)$ & $0(0.00)$ \\
\hline \multicolumn{5}{|l|}{ Overall } \\
\hline Radiotherapy & 996 & $27(0.03)$ & $49(0.05)$ & $4(0.004)$ \\
\hline Control & 1015 & $87(0.09)$ & $17(0.02)$ & $0(0.000)$ \\
\hline Risk difference $(95 \% \mathrm{Cl})$ & 2011 & ARR: $0.06(0.04-0.08)$ & $A R I_{\text {morbidity: }} 0.03(0.02-0.05)$ & $A R I_{\text {mortality: }} 0.004(0.000-0.008)$ \\
\hline
\end{tabular}

$A R I_{\text {morbidity }}=$ absolute risk increase in radiation-induced morbidity; $A R I_{\text {mortality }}=$ absolute risk increase in radiation-induced mortality; $A R R=$ absolute risk reduction in the development of local recurrences. 95\% Cl=95\% confidence interval. Bold represent the pooled estimates of the three studies described above (PORTEC-I, GOG99 and ASTEC/EN.5).

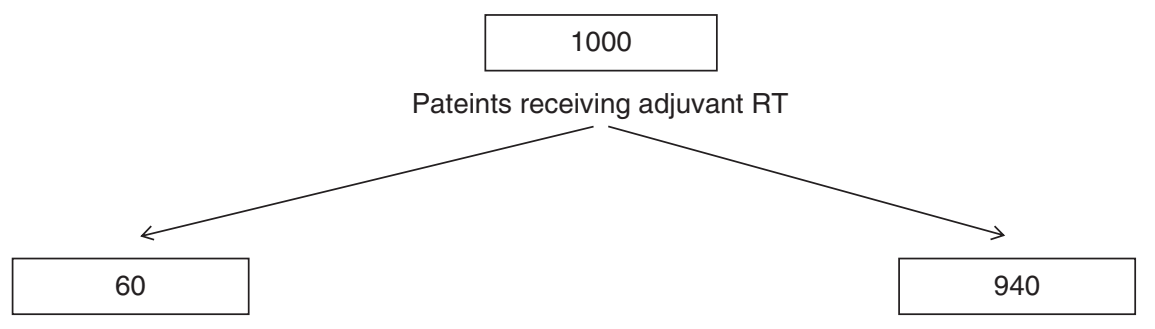

Beneficial: local reccurence prevented by $\mathrm{RT} *$

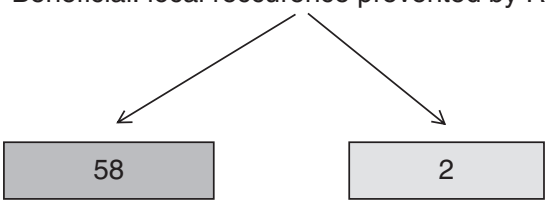

1. Full benefit, no harm:

Patients in whom RT prevented the development of a local recurrence and who do not suffer from severe complications due to RT
2. Benefit with harm: prevented the development of a local recurrence, but suffer from severe complications
Patients in whom RT
Not beneficial: local recurrence not prevented by $\mathrm{RT} *$

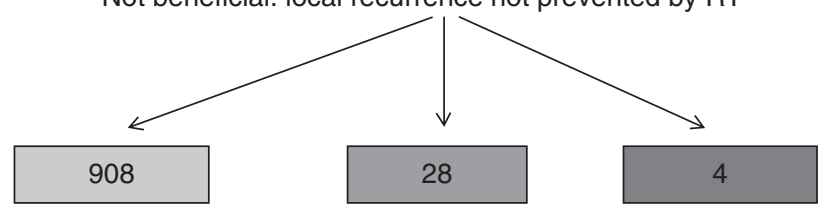

3. Neither benefit nor harm:

Patients in whom RT neither prevente a local recurrence nor induced severe complications
4. No benefit, but harm:

Patients in whom RT did not a prevent local recurrence and suffer from severe complications due to RT

Figure I Benefit and harm for a fictive cohort of 1000 irradiated patients in whom adjuvant RT is compared with no adjuvant treatment. *This figure displays the differences between adjuvant RT and no adjuvant therapy rather than absolute frequencies, that is the additional benefit in terms of local recurrences as prevented by adjuvant RT and the additional harm in terms of severe complications and deaths as induced by adjuvant RT.

(ASTEC/EN.5) (Creutzberg et al, 2000; Keys et al, 2004; the ASTEC/ EN.5 writing committee 2008, 2009). They comprised 2011 women, and relevant data on benefit and harm in the intervention and control groups could be extracted (Table 2).

All studies were of a randomised design, compared the same interventions (adjuvant pelvic external beam RT $v s$ no adjuvant therapy) and reported the required outcome measures (local recurrence rate and treatment-induced harm) on similar patients (intermediate-risk stage I endometrial cancer patients). In the ASTEC/EN.5 trial, brachytherapy was also allowed if the centre's policy was to offer it to all International Federation of Gynecology and Obstetrics (FIGO) stage I or IIA women irrespective of RT allocation. Similar proportions of women in the RT group as well as the control group received brachytherapy (52 and $51 \%$, respectively). Providing brachytherapy might lead to an under- estimation of the beneficial effect of adjuvant external RT and an overestimation of the risk of complications of adjuvant RT for stage I intermediate-risk endometrial cancer patients. However, sensitivity analyses showed that the numbers in the model hardly changed when only the PORTEC-1 and GOG 99 trials were taken into account (ARR 0.09, ARI morbidity 0.04 and ARI mortality 0.005). All in all, we decided that the data of these three studies could be used to calculate an overall ARR and ARI to complete our scheme.

Subsequently, the overall ARR and ARIs were combined by means of the equations in Table 1 to compose the five possible outcome groups. This is illustrated in a fictive cohort of 1000 women receiving adjuvant RT for their stage 1 intermediate-risk endometrial carcinoma (Figure 1). This shows that from a putative cohort of 1000 patients, adjuvant RT will prevent recurrences in 60 patients (groups 1 and 2), a majority of 908 patients will neither 
benefit nor suffer severe radiation-induced harm (group 3), but 28 patients will suffer severe complications due to adjuvant RT (group 4) and an expected four patients will die (group 5).

\section{DISCUSSION}

In this study, a scheme is presented to balance the benefit and harm of adjuvant therapy $v s$ no adjuvant therapy. To exemplify this scheme, we utilised the data on adjuvant pelvic external beam RT for intermediate-risk stage I endometrial carcinoma patients. The scheme readily provides an insight into the proportion of patients who will benefit from adjuvant therapy (some at the cost of complications) versus those who neither benefit nor contract complications, and those who do not benefit from adjuvant therapy but do contract severe complications, or even die.

It may be hard to imagine that providing an adjuvant therapy does not necessarily lead to the intended effect and can even harm our patients. However, as complications might harm patients who do not have any benefit from adjuvant therapy, we advocate the evidence as presented in this scheme to be communicated to them. This will enable patients to determine their preference regarding adjuvant therapy based on comprehensive information, which ultimately facilitates shared decision making (Charles et al, 1997; van Tol-Geerdink et al, 2008).

In our example of intermediate-risk stage I endometrial carcinoma patients, here is what one could communicate about adjuvant RT based on the insight provided by the completed scheme. The patient should first be informed about the risk of developing a local recurrence, that is about $10 \%$ according to our included studies. Providing adjuvant RT will not improve the patient's chances of survival, but does decrease the risk of developing a local recurrence. Along with communicating the probability of developing a local recurrence, one should try and explain to the patient what it is like to experience this. Subsequently, our scheme may be used by clinicians to explain that in a group of 1000 patients like them, if they would undergo postoperative RT, the majority (908) will neither benefit from RT in terms of prevented local recurrences, nor suffer from severe complications. The physical and mental discomfort of a local recurrence will be prevented in 60 patients, whereas serious, sometimes even life-threatening, complications from RT will occur in 28 patients, and an expected four patients will die. An explanation of what it would be for the patient to experience severe complications is needed, and it should be stressed that severe complications are included in the scheme (because these complications could cause them to reconsider having RT or not), but moderate and mild complications are not.

\section{REFERENCES}

Aalders J, Abeler V, Kolstad P, Onsrud M (1980) Postoperative external irradiation and prognostic parameters in stage I endometrial carcinoma: clinical and histopathologic study of 540 patients. Obstet Gynecol 56: 419-427

Bakx R, Emous M, Legemate DA, Zoetmulder FA, van TG, Bemelman WA, van Lanschot JJ (2006) Harm and benefits of short-term pre-operative radiotherapy in patients with resectable rectal carcinomas. Eur J Surg Oncol 32: $520-526$

Burger MP (2001) Management of stage 1 endometrial carcinoma. Postoperative radiotherapy is not justified in women with medium risk disease. BMJ 322: $568-569$

Charles C, Gafni A, Whelan T (1997) Shared decision-making in the medical encounter: what does it mean? (or it takes at least two to tango) Soc Sci Med 44: 681-692

Chassagne D, Sismondi P, Horiot JC, Sinistrero G, Bey P, Zola P, Pernot M, Gerbaulet A, Kunkler I, Michel G (1993) A glossary for reporting
By means of this scheme, benefit and harm of two adjuvant therapies, or an adjuvant therapy versus no adjuvant treatment, can be balanced. Although this provides a convenient and orderly overview, the scheme is of less value in case more adjuvant therapies are available. For example, for stage I intermediate-risk endometrial cancer patients, vaginal intracavitary brachytherapy is currently hypothesised to be superior to external RT in terms of morbidity and mortality due to its local rather than regional effect (http://www.trialregister.nl/trialreg/ admin/rctview.asp?TC $=332$ ). These two adjuvant therapies could be compared in a scheme as presented in this paper; however, the option of providing no adjuvant treatment cannot be incorporated in the same scheme. This leads to three separate schemes comparing: (1) external beam RT $v s$ no adjuvant therapy, (2) brachytherapy $v s$ no adjuvant therapy and (3) external beam RT $v s$ brachytherapy, which is far too complicated to gain a view of the situation.

As the development of local recurrences is nowadays considered to be the primary end-point in studies of adjuvant RT, we chose local recurrence rate as the primary end-point in our scheme as well. However, we realise that this is an intermediate end point as it does not include subsequent benefit and harm of therapy after the development of a recurrence. Salvage therapy includes surgery, external beam RT and intracavitary RT, and is experienced as physically extremely uncomfortable and mentally very stressful, causing a substantial decrease in quality of life. Survival after relapse is significantly better in patients who have not received adjuvant RT than in those who already did (3-year survival 51 vs $19 \%(P=0.004))$ (Creutzberg et al, 2003). However, this does not lead to a better overall survival in patients without adjuvant RT. In the scheme presented, it was impossible to incorporate the morbidity and mortality of treating a local recurrence by means of salvage therapy, although it is likely that the clinical bottom line would hardly change due to the great share of patients receiving neither benefit nor harm from adjuvant RT. When deciding about adjuvant RT, patients should therefore also be informed about the procedure of salvage therapy and the differences in survival after relapse.

The scheme as presented in this paper readily summarises the different possible treatment outcomes and can be of practical value for clinicians as well as for patients in (shared) decision making about adjuvant therapies. Moreover, it can be, and has already been in a few instances, generalised towards other interventions in other medical specialties, when pros and cons need to be weighed to make a treatment decision. Future research should reveal if clinicians as well as patients evaluate this scheme as a valuable tool to balance benefit and harm and whether it affects their final treatment decision. complications of treatment in gynecological cancers. Radiother Oncol 26: $195-202$

Creutzberg CL, van Putten WL, Koper PC, Lybeert ML, Jobsen JJ, WarlamRodenhuis CC, De Winter KA, Lutgens LC, van den Bergh AC, van de Steen-Banasik E, Beerman H, van Lent M (2000) Surgery and postoperative radiotherapy $v s$ surgery alone for patients with stage- 1 endometrial carcinoma: multicentre randomised trial. PORTEC Study Group. Post Operative Radiation Therapy in Endometrial Carcinoma. Lancet 355: $1404-1411$

Creutzberg CL, van Putten WL, Koper PC, Lybeert ML, Jobsen JJ, WarlamRodenhuis CC, De Winter KA, Lutgens LC, van den Bergh AC, van de Steen-Banasik E, Beerman H, van Lent M (2003) Survival after relapse in patients with endometrial cancer: results from a randomized trial. Gynecol Oncol 89: 201-209

Gigerenzer G, Edwards A (2003) Simple tools for understanding risks: from innumeracy to insight. $B M J$ 327: $741-744$ 
Hoffrage U, Lindsey S, Hertwig R, Gigerenzer G (2000) Medicine. Communicating statistical information. Science 290: 2261-2262

http://www.trialregister.nl/trialreg/admin/rctview.asp?TC $=332$. Internet. Last accessed: 01-30-2009

http://www.guideline.gov/summary/summary.aspx?doc_id $=9203 \& \mathrm{nbr}=$ 004957\&string $=$ endometrial+AND+cancer. Internet. Last accessed: 01-30-2009

Johnson N, Cornes P (2007) Survival and recurrent disease after postoperative radiotherapy for early endometrial cancer: systematic review and meta-analysis. BJOG 114: $1313-1320$

Keys HM, Roberts JA, Brunetto VL, Zaino RJ, Spirtos NM, Bloss JD, Pearlman A, Maiman MA, Bell JG (2004) A phase III trial of surgery with or without adjunctive external pelvic radiation therapy in intermediate risk endometrial adenocarcinoma: a Gynecologic Oncology Group study. Gynecol Oncol 92: 744-751

Kong A, Johnson N, Cornes P, Simera I, Collingwood M, Williams C, Kitchener H (2007) Adjuvant radiotherapy for stage I endometrial cancer. Cochrane Database Syst Rev 18(2): CD003916

Mancini GB, Schulzer M (1999) Reporting risks and benefits of therapy by use of the concepts of unqualified success and unmitigated failure: applications to highly cited trials in cardiovascular medicine. Circulation 99: $377-383$

Massel D (2003) The number needed to harm: is it too optimistic? Can J Cardiol 19: 1490 - 1492

McAlister FA, Straus SE, Guyatt GH, Haynes RB (2000) Users' guides to the medical literature: XX. Integrating research evidence with the care of the individual patient. Evidence-Based Medicine Working Group. JAMA 283: $2829-2836$

Nuovo J, Melnikow J, Chang D (2002) Reporting number needed to treat and absolute risk reduction in randomized controlled trials. JAMA 287: $2813-2814$
Pieterse AH, Stiggelbout AM, Baas-Thijssen MC, van de Velde CJ, Marijnen CA (2007) Benefit from preoperative radiotherapy in rectal cancer treatment: disease-free patients' and oncologists' preferences. Br J Cancer 97: $717-724$

Schulzer M, Mancini GB (1996) 'Unqualified success' and 'unmitigated failure': number-needed-to-treat-related concepts for assessing treatment efficacy in the presence of treatment-induced adverse events. Int $J$ Epidemiol 25: 704-712

Soderini A, Anchezar JP, Sardi JE (2003) Role of adjuvant radiotherapy (RT) in intermediate risk (1b G2-3-1C) endometrioid carcinoma (EC) after extended staging surgery (ESS). Preliminary reports of a randomised trial. Int J Gynecol Cancer Abstract P0147(suppl 1): 78

Straus SE (2002) Individualizing treatment decisions. The likelihood of being helped or harmed. Eval Health Prof 25: 210-224

The ASTEC/EN.5 writing committee 2008 (2009) Adjuvant external beam radiotherapy in the treatment of endometrial cancer (MRC ASTEC and NCIC CTG EN.5 randomised trials): pooled trial results, systematic review, and meta-analysis. The Lancet 373: 97-99

van Tol-Geerdink JJ, Leer JW, van Lin EN, Schimmel EC, Huizenga H, van Daal WA, Stalmeier PF (2008) Offering a treatment choice in the irradiation of prostate cancer leads to better informed and more active patients, without harm to well-being. Int J Radiat Oncol Biol Phys 70: $442-448$

van Tol-Geerdink JJ, Stalmeier PF, van Lin EN, Schimmel EC, Huizenga H, van Daal WA, Leer JW (2006) Do prostate cancer patients want to choose their own radiation treatment? Int J Radiat Oncol Biol Phys 66: $1105-1111$

Weigensberg IJ (1984) Preoperative radiation therapy in Stage I endometrial adenocarcinoma. II. Final report of a clinical trial. Cancer 53: $242-247$ 